\title{
Trapping of phase fronts and twisted spirals in periodically forced oscillatory media
}

\author{
Oliver Rudzick and Alexander S. Mikhailov
}

Abteilung Physikalische Chemie, Fritz-Haber-Institut der Max-Planck-Gesellschaft, Faradayweg 4-6, 14195 Berlin, http://www.fhi-berlin.mpg.de/complsys/

\section{Periodically forced oscillatory media}

The control of pattern formation is an important problem in engineering of spatially extended self-organized systems. A wide class of systems showing spontaneously formed waves and patterns can be summarized under the generic term "oscillatory media". They may be considered as a composition of a large number of coupled subsystems. The dynamics of each subsystem is oscillatory. Complex phenomena like the formation of patterns and waves or spatio-temporal chaos comes through the interaction between the subsystems. Despite such complex collective behavior the dynamics is very sensitive to feedback or to periodic forcing with frequencies close to an integer ratio of the oscillation frequency of the subsystems.

A well-known example is the Belouzov-Zhabotinsky-Reaction, involving the oxidation of an organic compound by bromate in acidic solution. It is a robust oscillatory reaction with striking color changes. Spatiotemporal wave behavior is exhibited in unstirred reaction mixtures $[1,2]$. For this reaction, application of global periodic forcing was shown to produce various cluster patterns $[3,4,5]$ and induce turbulent regimes [6].

Another example which has been extensively studied is the catalytic CO oxidation on $\mathrm{Pt}(110)$. The interplay between desorption and surface diffusion of $\mathrm{CO}$, reaction between the two adsorbed species, and an adsobate-driven structural change of the platinum surface can lead to oscillations of the CO and oxygen coverage [7]. In experiments where bulk oscillations were unstable and spatiotemporal chaos spontaneously developed, application of periodic forcing allowed to suppress chemical turbulence, produce intermittent regimes with cascades of amplitude defects, and generate oscillating cellular and labyrinthine patterns $[8,9]$. Recently, front explosions have been predicted under periodic forcing [10]. 

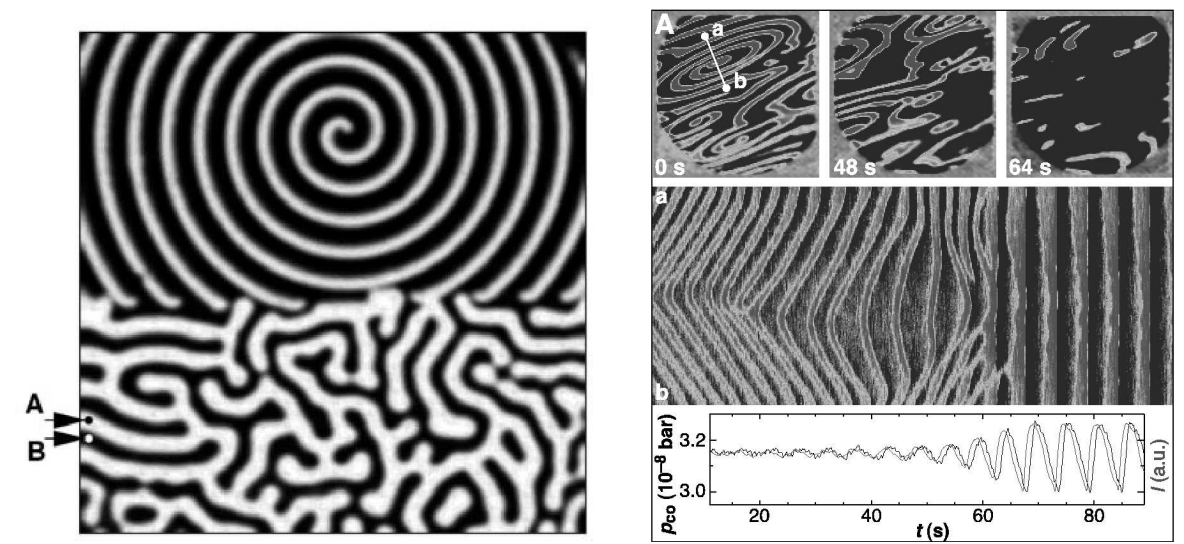

Fig. 1. Left: Effect of 2:1 resonant periodic forcing on the photosensitive BZreaction. A spiral wave is formed without forcing (upper part). Under the influence of illumination with light pulsed at twice the natural frequency of the reaction a labyrithine pattern appear.[3]. Right: Suppression of chemical turbulence in the catalytic $\mathrm{CO}$ oxidation on $\mathrm{Pt}(110)$ under global feedback. Upper row: subsequent PEEM images of the Pt surface illustrating the transition from spiral wave turbulence to homogeneous oscillations. Bright regions are mainly CO-covered. Middle row: Space-time diagram showing the evolution along the line AB. Bottom: Time series of the CO partial pressure[8].

\section{The forced complex Ginzburg-Landau equation}

The universal description of reaction-diffusion systems near a supercritical Hopf bifurcation is provided by the complex Ginzburg-Landau equation [11]. Action of global periodic forcing on the systems described by this equation has been first considered by Coullet and Emilsson [12, 13]. Under sufficiently strong resonant n:1 forcing, oscillations are entrained and stationary or traveling $2 \pi / n$-fronts become possible. The $2 \pi$ phase fronts for the $1: 1$ forcing are known as kinks (or phase slips). They represent traveling localized structures, because the states differing by the phase of $2 \pi$ are physically identical. Therefore, as noticed in Ref. [13], they bear similarity with pulses in excitable media. Traveling $\pi$-fronts under 2:1 forcing represent nonequilibrium Bloch walls [14]. Kinks and traveling Bloch walls are elementary wave patterns under forcing conditions. Instabilities of kinks lead to backfiring and development of intermittent regimes with reproduction of amplitude defects [15, 16, 17]. Transverse instabilities of nonequilibrium planar Bloch walls give origin to the Bloch turbulence [6]. In heterogeneous media near a Bloch-Ising transition, complex behavior due to reflections of Bloch waves on Ising domains has been found [18].

Under global resonant n:1 forcing, the complex Ginzburg-Landau equation (CGLE) for the slow complex oscillation amplitude $\eta$ is [12] 


$$
\dot{\eta}=(1+i \nu) \eta-(1+i \alpha)|\eta|^{2} \eta+(1+i \beta) \nabla^{2} \eta+B\left(\eta^{*}\right)^{n-1},
$$

where detuning $\nu=\omega_{0}-\omega_{e} / n$ is determined by the natural $\left(\omega_{0}\right)$ and forcing $\left(\omega_{e}\right)$ frequencies and $B$ is the forcing amplitude. Oscillations are entrained by forcing in the parameter region known as the Arnold tongue (Fig. 2 (a)). Inside this region, kinks $(n=1)$ and Bloch walls $(n=2)$ traveling at a constant velocity are possible (see $[12,15,16])$. Moreover, wave trains formed by periodic sequences of such phase fronts can also be observed there.

\section{Phase front propagation reversal}

Our attention is focused on the properties of periodic trains formed by kinks or traveling Bloch walls. Our analysis reveals that, depending on the parameters of the oscillatory medium and the spatial period of a train, it can undergo a reversal of its propagation direction [19]. We show how this phenomenon can be used to design traps for traveling kinks and Bloch walls. Furthermore, we find that a new kind of patterns - twisted rotated spiral waves - exist in oscillatory media under the conditions of front propagation reversal.

Any traveling phase front is characterized by its chirality: "right" if the phase increases after front propagation and "left" if it decreases after that. A similar definition can be accepted for traveling wave trains. It is convenient furthermore to define the front velocity $V$ in such a way that it is always positive $(V>0)$ if a front propagates to increase the oscillation phase and negative $(V<0)$ otherwise. With this convention, all "right" fronts move at a positive velocity, while the velocity of any "left" phase front is negative.

The velocity of an individual phase front is uniquely determined by the properties of the medium and the forcing parameters. For wave trains, it additionally depends on the spatial period $\lambda$ of a train. Figure $2 \mathrm{~b}$ shows dependences $V(\lambda)$ for two different values of the coefficient $\beta$, obtained by numerical continuation of wave train solutions of equation (1) with $n=1$. When $\beta=5.0$, velocity $V$ remains positive for all spatial periods. This means that both a solitary kink and any kink train in such a medium possess the "right" chirality. In contrast to this, kinks move at a positive velocity (and have the "right" chirality) only for sufficiently short spatial periods at $\beta=1.8$. At a critical spatial period $\lambda_{c}$, the propagation velocity of the train vanishes and $V(\lambda)<0$ when $\lambda>\lambda_{c}$. Thus, solitary kinks and kink trains with large periods have the opposite "left" chirality in the latter case.

To illustrate the difference in the properties of wave patterns in such two media, we consider the following example. Suppose that the local oscillation frequency is increased in the center of a one-dimensional medium. If periodic 1:1 forcing is applied, the local frequency increase can still be so large that oscillators in the central region are not entrained and perform autonomous oscillations. This region acts then as a pacemaker which periodically generates phase slips propagating away as a kink train with the "right" chirality . 


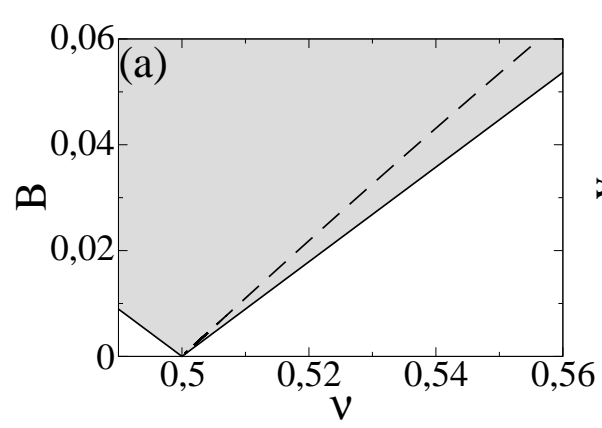

(c)

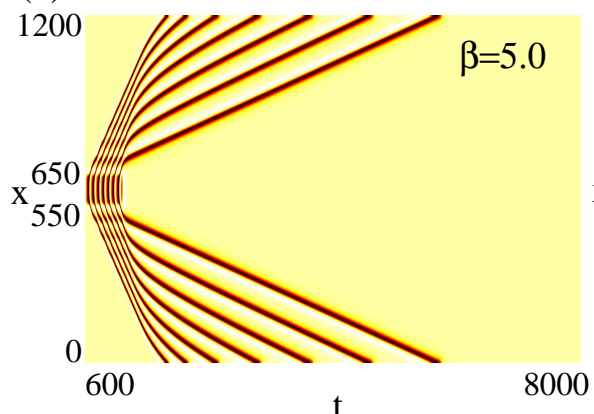

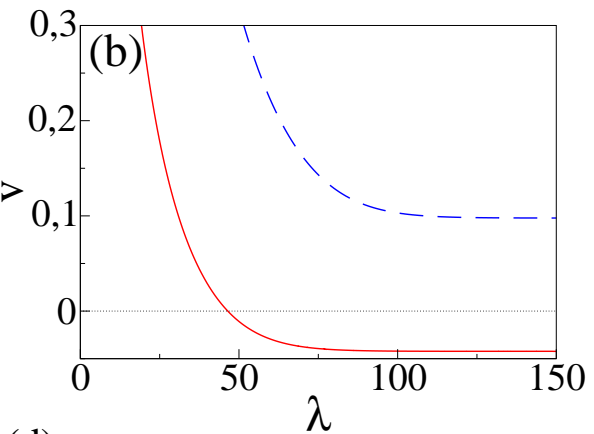

(d)

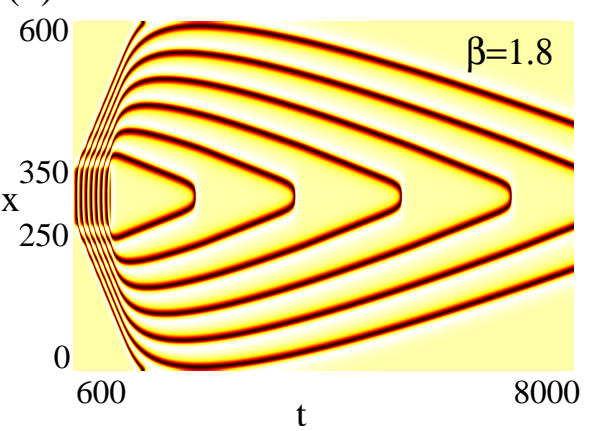

Fig. 2. Front propagation reversal $(n=1, \alpha=0.5)$. (a) Standing kinks are found along the dashed line $(\beta=1.8)$ in the Arnold tongue. (b) Dependences of velocity $V$ on spatial train period $\lambda$ for $\beta=1.8$ (solid line) and $\beta=5.0$ (dashed line); here $\nu=0.5525, B=0.053$. Space-time diagrams showing behavior of wave patterns after termination of a pacemaker in media with (c) $\beta=5.0$ and (d) $\beta=1.8$; the same other parameters as in part $\mathrm{b}$, local values of $\operatorname{Re} \eta(x, t)$ are shown in gray scale.

Suppose now that this heterogeneity is removed and the activity of the pacemaker is terminated. When $\beta=5.0$, generated kinks continue to move away from the center (Fig. 2c). The situation is however different, if $\beta=1.8$ (see Fig. 2d). As spatial intervals between the kinks get larger, they subsequently reverse their propagation direction and move into the central region where repeated annihilations take place. This is because single kinks and the kink trains with sufficiently large periods are characterized by the "left" chirality in this medium and propagate in such a way that the oscillation phase becomes decreased.

\section{Phase approximation}

For sufficiently small forcing amplitudes $B$, the train velocity $V(\lambda)$ can be analytically estimated. In this parameter region, the dynamics is approximately described [12] by the reduced equation for the local oscillation phase $\varphi$, 


$$
\dot{\varphi}=\nu-\alpha-B \sqrt{1+\alpha^{2}} \sin n \varphi+a(\nabla \varphi)^{2}+b \nabla^{2} \varphi,
$$

where $\varphi=\phi+\arctan \alpha$ and $\eta=\rho \exp (i \phi)$. For brevity, we have introduced here notations $b=1+\alpha \beta$ and $a=\alpha-\beta$. Note that such reduced phase description is justified, when $b>0$ so that uniform oscillations are modulationally (Benjamin-Feir) stable. The kinks exist for $B>B_{\mathrm{A}}$, where $B_{\mathrm{A}}(\nu)=|\nu-\alpha| / \sqrt{1+\alpha^{2}}$.

Applying the Cole-Hopf transformation $\varphi=(b / a) \ln u$, this phase dynamics equation is transformed to a simple form analogous to the equation for front propagation in one-component bistable media [20],

$$
\partial_{t} u=Q(u)+b \partial_{x x} u
$$

with the nonlinear function $Q(u)=(a / b) u\left[\nu-\alpha-B \sqrt{1+\alpha^{2}} \sin (n(b / a) \ln u)\right]$. The roots $u_{j}$ of equation $Q(u)=0$ under the condition $Q^{\prime}\left(u_{j}\right)<0$ correspond to stable uniform locked states of the system. Explicitely, we have

$$
u_{j}=\exp \left\{\frac{a}{n b}\left[2 \pi j+\arcsin \left(\frac{\alpha-\nu}{B \sqrt{1+\alpha^{2}}}\right)\right]\right\} .
$$

Although the system has an infinite sequence $j=1,2,3, \ldots$ of such roots, only $n$ of them represent physically different phase-locked states.

A front train with spatial period $\lambda$ is a solution of equation (3) satisfying periodicity conditions $\varphi(x+\lambda / n)=\varphi(x)+2 \pi / n$ (for $n>1$ one spatial period of the pattern consists of $n$ subsequent $2 \pi / n$-fronts). In terms of the variable $u$, these conditions take the form

$$
u(x+\lambda / n)=\exp (2 \pi a / n b) u(x) .
$$

Thus, the train solutions for $u$ are not periodic, but grow exponentially with $x$.

When propagation reversal occurs, a stationary train is possible. In the stationary case, equation (3) has the first integral $(1 / 2) b\left(\partial_{x} u\right)^{2}+W(u)=E$. Using the periodicity condition (5) and the property $W\left(u_{j+1}\right)=\exp (4 \pi a / n b) W\left(u_{j}\right)$, we find that $E=0$ for any stationary train. Thus, the wavelength $\lambda_{s t}$ of the stationary train is given by

$$
\lambda_{s t}=\int_{u_{j}}^{u_{j+1}} \sqrt{\frac{-b n^{2}}{2 W(u)} d u .}
$$

This result does not depend on the choice of the root $j$.

Solitary $2 \pi / n$ phase fronts are front solutions of equation (3), such that $u(x, t) \rightarrow u_{j}$ for $x \rightarrow-\infty$ and $u(x, t) \rightarrow u_{j \pm 1}$ for $x \rightarrow \infty$. They can be also viewed as a limit of a periodic train with $\lambda \rightarrow \infty$. According to equation (6), the wavelength of a stationary train diverges, if $W\left(u_{j}\right)=0$. Solving this equation, we find that stationary solitary $2 \pi / n$ fronts exist along the line $B=B_{s t}(\nu)$ given by 


$$
B_{s t}=\frac{1}{2 a}(\alpha-\nu) \sqrt{\frac{a^{2}+n^{2} b^{2}}{1+\alpha^{2}}} .
$$

Note that, in the phase approximation, the boundaries of the Arnold tongue are $B=B_{A}(\nu)$. The line $B=B_{s t}(\nu)$ is shown as the dashed line in Fig. 2a. Along this line, the reversal of the propagation direction of solitary phase fronts occurs. Phase fronts with the "right" chirality $(V>0)$ are found on the left side of this line, if $\beta>\alpha$.

As follows from (6), the condition for existence of stationary periodic trains is $W\left(u_{j}\right)<0$. This means that they are found inside the region of the Arnold tongue, lying between the line $B=B_{s t}(\nu)$ and the (nearest) boundary of the tongue (see Fig. 1a). Such a region always exists if $b>0$. For any given set of parameters, the wavelength of the stationary train can be computed by numerical evaluation of the integral in equation (6).

The above analysis shows that front propagation reversal occurs near any n:1 resonance. For $n=1$, stationary kinks (i.e., $2 \pi$-fronts) and periodic sequences of standing kinks are possible (the existence of stationary solitary kinks under global feedback conditions has previously been shown [15]). For $n=1$, stationary $\pi$-fronts represent standing Bloch walls or their periodic sequences. Such standing structures are different from Ising walls, because the oscillation amplitude does not vanish here.

\section{Trapping of phase fronts}

The wave propagation reversal can be induced by varying parameters of the medium. Most conveniently, this can be done by changing the coefficient $\beta$ in the CGLE, since this coefficient does not affect uniform oscillations and is only important for propagating waves.

The dependence of the wave propagation direction on the coefficient $\beta$ can be used to trap kinks and Bloch fronts. Such traps can be designed by creating spatial regions, where the coefficient $\beta$ is locally changed to reverse the propagation velocity. The left panel in Fig. 3 shows an example of a kink trap in the one-dimensional medium at the 1:1 resonance. The value of $\beta$ is decreased in the central region. No-flux boundary conditions are used in all our simulations. Initially, a rapid pacemaker operates at the left end of the medium. This pacemaker produces a kink train with a short spatial period. The train enters the modified central region and passes it with some deceleration. When the pacemaker is terminated, further kinks are not produced. However, the kinks inside the central modified region become trapped inside it and form a stationary pattern with a period corresponding to the velocity reversal. If the central heterogeneity is removed, the stored pulses would propagate out of it. The right panel in Fig. 3 demonstrates the trapping of Bloch fronts at the 2:1 resonance. The pacemaker at the left end of the medium produces a train of Bloch fronts. 

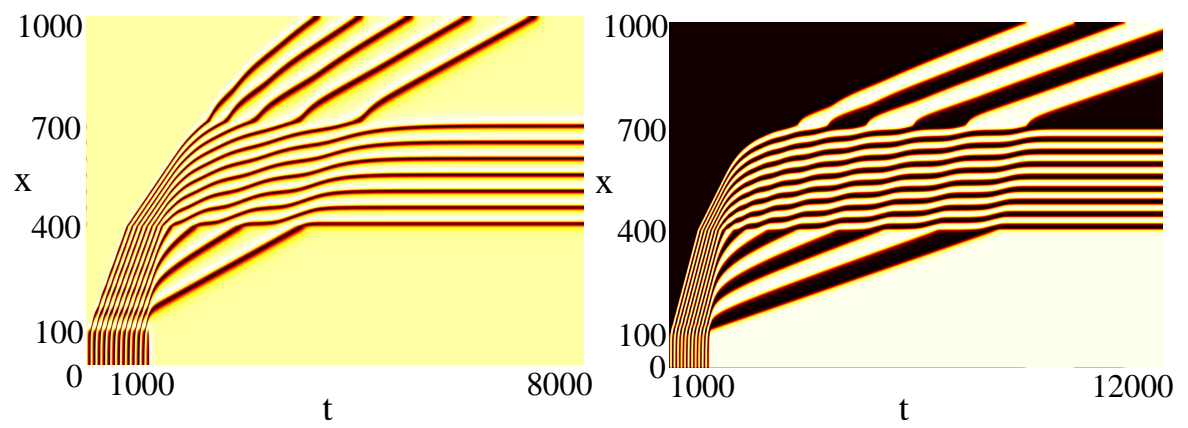

Fig. 3. Trapping of kinks and Bloch fronts in the $1-\mathrm{d}$ medium. The coefficient $\beta$ is set to $\beta=5$ and decreased to $\beta=1.8$ inside the central region of width 300 . Left: Kink trap under the 1:1 resonance, the same parameters as in Fig. 1b. Right: Bloch front trap under the $2: 1$ resonance $(B=0.061$, the same other parameters as in Fig. 1b).


Fig. 4. Trapping of kinks and Bloch fronts in the two-dimensional medium. The coefficient $\beta$ is set to $\beta=5$ and decreased to $\beta=1.8$ in the rectangular central region. The medium parameters are the same as in Fig. 2. The system size is $1000 \times 1000$. Upper panel: Kink trap in 1:1 resonance. The snapshots of the spatial distribution of Re $\eta$ are taken at $t=6000, t=19000$ and $t=32000$. Lower panel: Bloch wave trap in 2:1 resonance. Snapshots the spatial distribution of $\operatorname{Re} \eta$ at $t=2000, t=16000$ and $t=46800$. $(B=0.061)$ 
Similar traps for kinks and Bloch fronts can also be constructed in twodimensional media. The upper panel of Fig. 4 (see also video 1 [21]) shows a series of snapshots where trapping of kinks by a central modified region is seen. A pacemaker in the lower left corner emits a kink train with a short spatial period. The first snapshot shows the kink train passing the rectangularshaped inhomogeneity. Inside the inhomogeneity, the velocity of the kinks is decreased, resulting in a delay of the kinks at the upper right corner of the rectangle. In the second snapshot, the rear end of the kink train has reached the lower left boundary of the inhomogeneity. The kinks inside the inhomogeneity close to this boundary can no longer propagate and the kinks outside propagate around the central region. This leads to the formation of ring-shaped kinks in the upper right part of the inhomogeneity. As long as new kinks arrive, these rings collapse. When the kink train has passed the inhomogeneity, the stored kinks form stationary ring-shaped structures. In the lower panel of Fig. 4 (see also video 2 [21]), trapping of traveling Bloch fronts at the 2:1 resonance is demonstrated. It proceeds similar to the respective process for the kinks near the 1:1 resonance. In our simulations, wave traps with various sizes and with complicated geometries could be created.

In the above discussion, we assumed that the condition $\beta>\alpha$ was satisfied. If the opposite condition $\beta<\alpha$ holds, waves in the unforced CGLE have negative dispersion (see, e.g., [22]). In this case, inwardly rotating spirals ("antispirals") and inwardly propagating target patterns are possible. In such media, reversal of front propagation also takes place and the wavelength of a stationary train is again given by equation (6). However, the line $B=B_{s t}(\nu)$ lies now on the left side of the Arnold tongue and stationary front trains are found in the region between this line and the left tongue boundary.

\section{Twisted spirals}

A special effect, related to kink propagation reversal, is the formation of twisted spirals near the 1:1 resonance in two-dimensional media (Fig. 5a). The central and outer parts in such a spiral are wound in opposite directions. These structures are stable, they are observed in numerical simulations starting with various initial conditions. A twisted spiral rigidly rotates as a whole, retaining its shape. In Fig. 5b, three subsequent snapshots of the spiral, separated by a third of the rotation period each, are superimposed (see also video $3[21]$ ). We see that the instantaneous rotation center does not coincide with the location of the spiral tip. Instead, the oppositely wound central part of the spiral is steadily rotating. Thus, this regime can also be characterized as a kind of meandering. Qualitatively, the development of twisted spirals can be understood by noticing that the waves are tightly wound near the center and, therefore, their propagation direction should be reversed there. In the displayed simulation, the medium was characterized by negative dispersion $(\beta<\alpha)$. Similar behavior has, however, been found by us in the simulations 

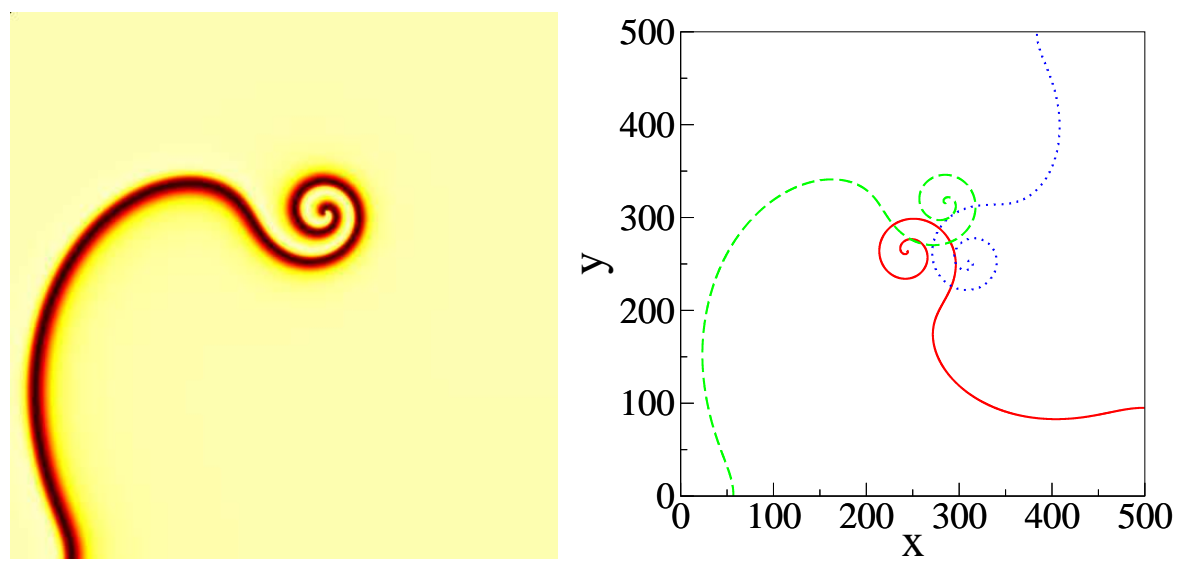

Fig. 5. Rotation of a twisted spiral. Left: Spatial distribution of Re $\eta$. Right: Position of the spiral at times $t=0$ (solid), $t=340$ (dashed), $t=680$ (dotted). Parameters are $\alpha=4.19, \beta=0.992, \nu=3.9895, B=0.0455$, the system size is $500 \times 500$. Numerical integration using the explicit Euler scheme with $\Delta x=0.2$ and $\Delta t=$ 0.0025 .

for the media with positive wave dispersion $(\beta>\alpha)[19]$. By changing the forcing intensity and frequency, winding and unwinding of the central part of the spiral can be controlled.

Our theoretical study has shown that, applying periodic forcing, one can induce propagation reversal of kinks, Bloch walls and $2 \pi / n$ phase fronts for higher resonances with $n>2$. Using this effect, traps for propagating kinks and other phase fronts can be designed by creating appropriate heterogeneities in the medium. In our simulations, such heterogeneities were introduced by spatial variation of the coefficient $\beta$ in the CGLE, but similar effects can be achieved by varying other parameters of the medium or by applying inhomogeneous forcing. We have also shown that, in uniform media with 1:1 forcing, steadily rotating twisted spirals can develop. Though our results have been obtained only for the CGLE, we expect that they should be characterictic for a class of media where oscillations are not strongly relaxational. Our simulations using a realistic model of the catalytic surface reaction of $\mathrm{CO}$ oxidation on platinum have shown that the wave propagation reversal under periodic forcing takes place near a supercritical Hopf bifurcation in this reaction and that the wave traps can be constructed there [19]. Another experimental system where the predictions of our theory can be tested is the oscillatory photosensitive Belousov-Zhabotinsky reaction.

\section{References}

1. A. N. Zaikin and A. M. Zhabotinsky. Concentration wave propagation in twodimensional liquid-phase self-oscillating system. Nature, 225:535, 1970. 
2. Arthur T. Winfree. Scroll-shaped waves of chemical activity in three dimensions. Science, 7:937-939, 1973.

3. V. Petrov, Q. Ouyang, and H. L. Swinney. Resonant pattern formation in a chemical system. Nature, 388:655 - 657, 1997.

4. A. L. Lin, M. Bertram, K. Martinez, H. L. Swinney, A. Ardelea, and G. F. Carey. Resonant phase patterns in a reaction-diffusion system. Phys. Rev. Lett., 84:4240, 2000.

5. V. K. Vanag, A. M. Zhabotinsky, and I. R. Epstein. Oscillatory Clusters in the Periodically Illuminated, Spatially Extended Belousov-Zhabotinsky Reaction. Phys. Rev. Lett., 86:552, 2001.

6. B. Marts, A. Hagberg, E. Meron, and A. L. Lin. Bloch-Front turbulence in a periodically forced Belousov-Zhabotinsky reaction. Phys. Rev. Lett., 93:108305, 2004.

7. K. Krischer, M. Eiswirth, and G. Ertl. Oscillatory CO oxidation on $\operatorname{Pt}(110)$ : modelling of temporal self-organization. J. Chem. Phys., 96(12):9161-9172, 1992.

8. M. Kim, M. Bertram, M. Pollmann, A. v. Oertzen, A. S. Mikhailov, H. H. Rotermund, and G. Ertl. Controlling chemical turbulence by global delayed feedback: pattern formation in catalytic co oxidation reaction on $\mathrm{pt}(110)$. Science, 292:1357-1360, 2001.

9. M. Bertram, C. Beta, H. H. Rotermund, and G. Ertl. Complex patterns in a periodically forced surface reaction. J. Phys. Chem. B, 107:9610, 2003.

10. J. Davidsen, A. S. Mikhailov, and R. Kapral. Front explosion in a periodically forced surface reaction. Phys. Rev. E, 72:046214, 2005.

11. I. S. Aranson and L. Kramer. The world of the complex Ginzburg-Landau equation. Rev. Mod. Phys., 74(1):99-143, 2002.

12. P. Coullet and K. Emilsson. Strong resonances of spatially distributed oscillators: a laboratory to study patterns and defects. Physica D, 61:119-131, 1992.

13. P. Coullet and K. Emilsson. Pattern formation in the strong resonant forcing of spatially distributed oscillators. Physica A, 188:190-200, 1992.

14. P. Coullet, J. Lega, B. Houchmanzadeh, and J. Lajzerowicz. Breaking chirality in nonequilibrium systems. Phys. Rev. Lett., 65:1352, 1990.

15. F. Mertens, R. Imbihl, and A. S. Mikhailov. Breakdown of global coupling in oscillatory chemical reactions. J. Chem. Phys., 99(11):8668-8671, 1993.

16. H. Chaté, A. Pikovsky, and O. Rudzick. Forcing oscillatory media: Phase kinks vs. synchronization. Physica D, 131(1-4):17-30, 1999.

17. M. Argentina, O. Rudzick, and M. G. Velarde. On the Back-firing instability. Chaos, 14(3):777 - 783, 2004.

18. C. Hemming and R. Kapral. Phase front dynamics in inhomogeneously forced oscillatory systems. Physica D, 306:199-210, 2002.

19. O. Rudzick and A. S. Mikhailov. unpublished.

20. A. S. Mikhailov. Foundations of Synergetics I. Distributed Active Systems. Springer, Berlin, 2nd ed., 1994.

21. Supporting online material can be found at http://www.fhi-berlin.mpg.de/complsys/cs_videos/twisted_spirals/.

22. M. Stich and A. S. Mikhailov. Complex pacemakers and wave sinks in heterogeneous oscillatory chemical systems. Z. Phys. Chem., 216:521-533, 2002. 